\title{
Systems medicine for the delivery of better healthcare Services- International Union for Physical and Engineering Sciences (IUPESM) perspective
}

\author{
Kin Yin Cheung ${ }^{1}$
}

Received: 1 December 2016 / Accepted: 6 December 2016 / Published online: 28 December 2016

(C) IUPESM and Springer-Verlag Berlin Heidelberg 2016

Medicine has ever been evolving in accordance with the clinical needs and the knowledge and philosophical attitude of the healthcare practitioners and service providers and at the same time influenced by geographical specific social, economic, political and environmental factors. The process is primarily aimed at improving human health through better access to quality medicine. One of the key indicators for quality medicine is clinical outcome, which is often determined by how early and accurate a disease is diagnosed and its staging determined and the efficacy of the clinical modalities used in treatment and post-treatment management of the patient. Biomedical engineers and medical physicists have played an important role in the development and operation of global healthcare services. One of their important contributions is in the research and development of better and more effective medical technologies in meeting clinical needs. They have close collaboration with healthcare professionals and medical scientists in development of the better tools and equipment to solve clinical challenges.

A major limitation in today's medicine is that the uniqueness of the biological properties and conditions of the individual patients are not properly accounted for when making disease diagnosis or medical intervention. The large variation in patient response to treatments observed in the clinics, particularly in cancer patients, indicates that current medical technologies and the associated physical and chemical interventional procedures alone cannot resolve all the clinical

This article is part of the Topical collection on Systems Medicine

Kin Yin Cheung

kycheung@hksh.com

1 Medical Physics \& Research Department, Hong Kong Sanatorium \& Hospital, Happy Valley, Hong Kong problems satisfactorily. A better understanding of the biology of the diseases at the genetic and DNA level is essentially required in order to be able to develop the optimal preventive and therapeutic modalities.

Current biological models are considered inadequate in fully explaining the multi-factorial nature and complex behavior of many human disorders and in predicting their response to the various forms of medical intervention. There is a need to develop a more systematic biological approach to address clinical problems. Systems medicine [1-3] is one of the approaches or concepts based on knowledge of systems biology being developed to guide treatment and prevention of diseases. Systems biology involves the analysis and understanding of the cellular and pathogenetic mechanisms and pattern of disease progression and remission, treatment responses and adverse effects at the individual patient level. The approach is aimed at providing the biological foundation or model needed by medical professionals for interpretation of biological and physical data collected from clinical investigations of the individual patients for making early disease diagnosis well before appearance of any symptoms or physical abnormalities. The biological information can also be used to guide any needed medical intervention and to predict and optimize treatment outcome on a personalized basis. Systems medicine, when realized will likely make a paradigm shift in medical practice and significant changes in the framework of global healthcare systems.

Development of systems medicine from concept to reality is a monumental task. This may involve several phases of development and key milestones on the road map may include the following.

(a) Development of the mathematical and computational models to simulate and analysis the genetic landscape of disease specific biological systems and dynamics of disease development process for different diseases 
(b) Translational research in application of systems biology model to systems medicine covering a comprehensive range of complex diseases.

(c) Research and development of the appropriate analytical, statistical, diagnostic and therapeutic tools and technologies for feasibility study and clinical testing.

(d) Clinical trials to verify the safety and efficacy of different approaches in systems medicine prior to large scale implementation healthcare services.

The development of systems medicine is essentially building a new foundation and infrastructure in medicine for delivery of more effective healthcare services. This demands a tremendous amount of resources such as finance, expertise, technologies, and associated facilities. It also requires the concerted efforts of healthcare professionals of different clinical specialties as well as scientific and engineering professionals of different disciplines contributing from different national and international institutions.

Much pioneering work along the line of systems medicine has already been carried out by medical scientists and engineers. DNA sequencing is one of the promising approaches or building blocks in development of systems medicine. The successful development of next-generation sequencing (NGS) technology [4-7] is a step forward in search of the linkage between human disorders and genetic defects arising from DNA mutation. The availability of supercomputers in handling the big data analysis of the NGS raw data of DNA/ RNA extracted from patient biological samples is making the technique feasible as a routine molecular pathological test. DNA sequencing has been applied to early detection of human disorders such as cancers and Downs Syndrome even before the patient is born. The technique is being used to test the sensitivity of normal and abnormal cells to therapeutic agents and the results can potentially be used for guiding treatment design such as dose prescription. The technique can be extended to study the genetic landscape and to evaluate the properties of biological defects of other diseases and health problems. The finding can potentially be used to develop new targeting therapeutic modalities and agents or to improve the efficacy of existing diagnostic and therapeutic modalities.

Research and development of systems medicine is a challenging, lengthy and costly evolution process. Biomedical engineers and medical physicists have been and will continue to play an important role in the process. They contribute to the research and development of appropriate medical technologies needed for development and implementation of systems medicine. As an umbrella organization for biomedical engineers and medical physicists, the International Union for Physical and Engineering Sciences in Medicine (IUPESM) brings together the two streams of professionals for mutual support and collaboration. The IUPESM also provides a scientific platform by organizing the World Congress on Medical Physics and Biomedical Engineering and publishing its official scientific journal, Heath and Technology to facilitate the interaction and networking between biomedical engineers and medical physicists with other professionals of different disciplines for exchange of ideas, research findings, and scientific and technological information. This in turn can foster interdisciplinary collaboration and stimulate the development of new concept and innovation in clinical research and development and contribute to the evolution of medicine.

\section{Compliance with ethical standards}

\section{Conflict of interest No conflict of interest.}

Funding There is no funding source.

Ethical approval This article does not contain any studies with human participants or animals performed by any of the authors.

Informed consent Not applicable.

\section{References}

1. Kyriakopoulou C, Mulligan B. Report on European Commission, DG Research, Directorate of Health Workshop on "From Systems Biology to Systems Medicine", Brussels. 2010.

2. Auffray C, Chen Z, Hood L. Systems Medicine: the future of medical genomics and healthcare. Genome Med. 2009;1(1):2.

3. Clermont G, Auffray C, Moreau Y, Rocke DM, Dalevi D, Dubhashi $\mathrm{D}$, et al. Bridging the gap between systems biology and medicine. Genome Med. 2009;1(9):88.

4. Tinhofer I, Niehr F, Konschak R, Liebs S, Munzm M, Stenzinger A, et al. Next-generation sequencing: hype and hope for development of personalized radiation therapy? Radiat Oncol. 2015;10:183.

5. Fiorentino F, Biricik A, Bono S, Spizzichino L, Cotroneo E, Cottone G, et al. Development and validation of a next-generation sequencingbased protocol for 24-chromosome aneuploidy screening of embryos. Fertil Steril. 2014;101(5):1375-82.

6. Kwong A, Shin VY, Au CH, Law FB, Ho DN, Ip BK, et al. Detection of germline mutation in hereditary breast and/or ovarian cancers by next-generation sequencing on a four-gene panel. J. Mol Diagn. 2016;18(4):580-94.

7. $\mathrm{CH}$ A et al. Clinical evaluation of panel testing by next-generation sequencing for gene mutations in myeloid neoplasms. Diagn Pathol. 2016;11:11. 